\title{
The Krull-Gabriel Dimension of Cycle-Finite Artin Algebras
}

\author{
Andrzej Skowroński ${ }^{1}$
}

Received: 18 May 2015 / Accepted: 18 August 2015 / Published online: 2 September 2015

(C) The Author(s) 2015. This article is published with open access at Springerlink.com

\begin{abstract}
We determine the Krull-Gabriel dimension of the cycle-finite categories of finitely generated modules over artin algebras and derive some consequences.
\end{abstract}

Keywords Krull-Gabriel dimension · Infinite radical · Auslander-Reiten quiver · Cycle-finite algebra

Mathematics Subject Classification (2010) $16 \mathrm{G} 10 \cdot 16 \mathrm{G} 60 \cdot 16 \mathrm{G} 70$

\section{Introduction and the Main Results}

Throughout the paper, by an algebra we mean an artin algebra over a commutative artin ring $K$. For an algebra $A$, we denote by $\operatorname{Mod} A$ the category of right $A$-modules, by $\bmod A$ the category of finitely generated right $A$-modules, and by ind $A$ the full subcategory of $\bmod A$ formed by the indecomposable modules. The $\operatorname{radical} \operatorname{rad}_{A}$ of $\bmod A$ is the ideal generated by all nonisomorphisms between modules in ind $A$. Then the infinite $\operatorname{radical} \operatorname{rad}_{A}^{\infty}$ of $\bmod A$ is the intersection of all powers $\operatorname{rad}_{A}^{i}, i \geq 1$, of $\operatorname{rad} A$. By a result of Auslander [3], $\operatorname{rad}_{A}^{\infty}=0$ if and only if $A$ is of finite representation type, that is, there are in ind $A$ only finite many modules up to isomorphism. On the other hand, if $A$ is of infinite representation type then $\left(\operatorname{rad}_{A}^{\infty}\right)^{2} \neq 0$, by a result proved in [13]. Moreover, we denote by $\Gamma_{A}$ the Auslander-Reiten quiver [6] of $A$ and by $\tau_{A}$ the Auslander-Reiten translation $D$ Tr. We do not distinguish between a module $X$ in ind $A$ and the corresponding vertex $\{X\}$ in $\Gamma_{A}$.

Presented by Jon F. Carlson.

Andrzej Skowroński

skowron@mat.uni.torun.pl

1 Faculty of Mathematics and Computer Science, Nicolaus Copernicus University,

Chopina 12/18, 87-100 Toruń, Poland 
A prominent role in the representation theory of algebras is played by cycles of indecomposable modules. Recall that a cycle in ind $A$ is a sequence

$$
M_{0} \stackrel{f_{1}}{\rightarrow} M_{1} \rightarrow \cdots \rightarrow M_{r-1} \stackrel{f_{r}}{\rightarrow} M_{r}=M_{0}
$$

of nonzero nonisomorphisms in ind $A$ [49], and such a cycle is said to be finite if the homomorphisms $f_{1}, \ldots, f_{r}$ do not belong to $\operatorname{rad}_{A}^{\infty}$ (see [1, 2]). Following Ringel [49], a module $M$ in ind $A$ which does not lie on a cycle in ind $A$ is said to be directing. It has been proved independently in [39] and [56] that the Auslander-Reiten quiver $\Gamma_{A}$ of an algebra $A$ admits at most finitely many $\tau_{A}$-orbits containing directing modules. Moreover, if all modules in ind $A$ are directing then $A$ is of finite representation type [49]. Following [1, 2], by a cyclefinite algebra we mean an algebra $A$ for which all cycles in ind $A$ are finite. The class of cycle-finite algebras is wide and contains the following distinguished classes of algebras: the algebras of finite representation type, the tame tilted algebras [24, 49], the tame double tilted algebras [46], the tame generalized double tilted algebras [47], the tubular algebras [49, 50], the tame quasi-tilted algebras [63], the tame generalized multicoil algebras [36], and the strongly simply connected algebras of polynomial growths [61]. Moreover, frequently interesting algebras admit Galois coverings by cycle-finite locally bounded categories, and applying covering techniques we may reduce their representation theory to that for the corresponding cycle-finite algebras (see $[1,16,38,54,62,64,66])$. The study of cycle-finite algebras has attracted much attention. We refer to [9, 10, 32-34, 37, 58-60, 67, 68] for some general results on cycle-finite algebras and their module categories.

Let $A$ be an algebra. We denote by $\mathcal{F}(A)$ the category of all finitely presented contravariant functors from $\bmod A$ to the category $\mathcal{A} b$ of abelian groups. The category $\mathcal{F}(A)$ was intensively studied over the last 40 years, and is considered to be one of the important topics of the modern representation theory of algebras. It is a hard problem to describe the category $\mathcal{F}(A)$ even if the category $\bmod A$ is well understood. A natural approach to study the structure of $\mathcal{F}(A)$ is via the associated Krull-Gabriel filtration

$$
0=\mathcal{F}(A)_{-1} \subseteq \mathcal{F}(A)_{0} \subseteq \mathcal{F}(A)_{1} \subseteq \cdots \subseteq \mathcal{F}(A)_{n-1} \subseteq \mathcal{F}(A)_{n} \subseteq \cdots
$$

of $\mathcal{F}(A)$ by Serre subcategories, where, for each $n \in \mathbb{N}, \mathcal{F}(A)_{n}$ is the subcategory of all functors $F$ in $\mathcal{F}(A)$ which become of finite length in the quotient category $\mathcal{F}(A) / \mathcal{F}(A)_{n-1}$ $[18,40]$. Following Geigle [22], we define $K G(A)=\min \left\{n \in \mathbb{N} \mid \mathcal{F}(A)_{n}=\mathcal{F}(A)\right\}$ if such a minimum exists, and set $K G(A)=\infty$ if it is not the case. Then $K G(A)$ is called the KrullGabriel dimension of $A$. We note that it is a finitely presented version of a definition due to Gabriel [21, IV.1]. The interest in the Krull-Gabriel dimension $K G(A)$ is motivated by the fact that the above filtration of $\mathcal{F}(A)$ leads to a hierarchy of exact sequences in $\bmod A$, where the almost split sequences form the lowest level (see [22]). Auslander proved that $K G(A)=0$ if and only if $A$ is of finite representation type (see [4, Corollary 3.14]). It was shown in $[26,11.4]$ that there exists no algebra $A$ with $K G(A)=1$. Further, $K G(A)=\infty$ for every wild hereditary algebra [7] and $K G(A)=2$ for any tame hereditary algebra $[22,4.3]$. There exist also tame algebras with infinite Krull-Gabriel dimension [23, 4.1]. Finally, it was shown independently in [12] and [52] that for any natural number $n \geq 2$ there exists a special biserial algebra (over an algebraically closed field $K$ ) with Krull-Gabriel dimension $n$. There is a strong relation between the Krull-Gabriel dimension $K G(A)$ of an algebra $A$ and the transfinite powers $\operatorname{rad}_{A}^{\alpha}$ of the $\operatorname{radical} \operatorname{rad}_{A}$ of $\bmod A$ (see [27, 5153]). In particular, Krause proved in [27, Corollary 8.14] that, if an algebra $A$ has finite Krull-Gabriel dimension $K G(A)=n$, then $\operatorname{rad}_{A}^{\omega(n+1)}=0$, where $\omega$ is the first limit number. 
The following general conjecture was posed in [53].

Conjecture 1.1 (Schröer) Let $n \geq 2$ be a natural number and $A$ an algebra. Then $K G(A)=n$ if and only if $\operatorname{rad}_{A}^{\omega(n-1)} \neq 0$ and $\operatorname{rad}_{A}^{\omega n}=0$.

In particular, for $n=2$, we have the following special case of Schröer's conjecture: for an algebra $A$ of infinite representation,

$K G(A)=2$ if and only if $\bigcap_{m \geq 1}\left(\operatorname{rad}_{A}^{\infty}\right)^{m}=0$.

It has been confirmed for the following classes of algebras: the tilted algebras of Euclidean type [22, 23], the algebras stably equivalent to tame hereditary algebras [23], the algebras with directing indecomposable projective modules [65], the enveloping algebras of restricted Lie algebras [19] (more generally, the infinitesimal group schemes [20]) in odd characteristic, the strongly simply connected algebras [61, 69], the 1-domestic string algebras [44, 45, 51], and recently the tame generalized multicoil algebras [31].

The following main result of the paper provides a characterization of cycle-finite algebras with finite Krull-Gabriel dimension, and confirms the special case of Schröer's conjecture.

Theorem 1.2 Let A be a cycle-finite algebra of infinite representation type. The following statements are equivalent:

(i) $K G(A)<\infty$.

(ii) $K G(A)=2$.

(iii) $\bigcap_{m \geq 1}\left(\operatorname{rad}_{A}^{\infty}\right)^{m}=0$.

(iv) $\operatorname{rad}_{A}^{\infty}$ is nilpotent.

(v) All but finitely many components of $\Gamma_{A}$ are stable tubes of rank one.

(vi) A does not admit a tubular quotient algebra.

An important tool in study of the Krull-Gabriel dimension of an algebra $A$ is the Ziegler spectrum $\mathrm{Zg}_{A}$ of $A$, introduced by Ziegler in his paper [71] on the model theory of modules. The Ziegler spectrum $\mathrm{Zg}_{A}$ is a topological space whose points are the isomorphism classes of indecomposable pure-injective modules in Mod $A$. Moreover, one associates to $A$ the Cantor-Bendixson rank $\mathrm{CB}(A)$ which measures the degree of isolation of points in the Ziegler spectrum $\mathrm{Zg}_{A}$. As was noted by Prest [43], for an artin algebra $A$, the isolated points of $\mathrm{Zg}_{A}$ are exactly the modules from ind $A$. In particular, one obtains that $\mathrm{CB}(A)=0$ if and only if $A$ is of finite representation type. Further, it has been proved by Ziegler in [71] that if $K G(A)=n<\infty$ then $\mathrm{CB}(A)=n$. Moreover, if $A$ is countable and $\mathrm{CB}(A)=n<\infty$, then $K G(A)=n$. We refer to [41, 42] for model theoretic, topological and geometric aspects of the Ziegler spectrum of an algebra.

The following corollary is a direct consequence of Theorem 1.2 and the Ziegler's result.

Corollary 1.3 Let $A$ be a cycle-finite algebra of infinite representation type and without a tubular quotient algebra. Then $\mathrm{CB}(A)=2$.

From the remarkable Tame and Wild Theorem of Drozd [17] (see also [14]) the class of finite dimensional algebras over an algebraically closed field $K$ may be divided into two disjoint classes. The first class is formed by the tame algebras for which the indecomposable modules occur in each dimension in a finite number of discrete and a finite number of one-parameter families. The second class is formed by the wild algebras whose representation theory comprises the representation theories of all finite dimensional algebras over $K$. 
Accordingly, we may realistically hope to classify the indecomposable finite dimensional modules only for the tame algebras. More precisely, a finite dimensional $K$-algebra $A$ over an algebraically closed field $K$ is called tame if for any dimension $d$, there exists a finite number of $K[x]$-A-bimodules $M_{i}, 1 \leq i \leq n_{d}$, which are free of finite rank as left modules over the polynomial algebra $K[x]$ in one variable and all but finitely many isomorphism classes of modules in ind $A$ of dimension $d$ are of the form $K[x] /(x-\lambda) \otimes_{K[x]} M_{i}$ for some $\lambda \in K$ and some $i$. Moreover, let $\mu_{A}(d)$ be the least number of $K[x]$ - $A$-bimodules satisfying the above condition for $d$. Then $A$ is said to be domestic if there exists a positive integer $m$ such that $\mu_{A}(d) \leq m$ for any $d \geq 1$ (see [15], [48], [55]). We also note that $A$ is of finite representation type if and only if $A$ is domestic with $\mu_{A}(d)=0$ for any $d \geq 1$ (by the validity of the second Brauer-Thrall Conjecture [8, 11]).

We have the following conjecture (see [53, p.420]):

Conjecture 1.4 (Prest) A finite dimensional algebra A over an algebraically closed field $K$ is domestic if and only if $K G(A)<\infty$.

The following direct consequence of Theorem 1.2 and [60, Theorem 5.1] confirms this conjecture for the cycle-finite algebras.

Corollary 1.5 Let $A$ be a finite dimensional cycle-finite algebra over an algebraically closed field $K$. Then $A$ is domestic if and only if $K G(A)<\infty$.

In the course of our proof of Theorem 1.2 we establish also the following result on the structure of module categories of cycle-finite algebras of finite Krull-Gabriel dimension.

Theorem 1.6 Let $A$ be a cycle-finite algebra of infinite representation type and without a tubular quotient algebra. Then there exist tame concealed quotient algebras $C_{1}, \ldots, C_{m}$ of $A$ and domestic generalized multicoil enlargements $B_{1}, \ldots, B_{m}$ of $C_{1}, \ldots, C_{m}$, respectively, such that the following statements hold:

(i) $B_{1}, \ldots, B_{m}$ are quotient algebras of $A$.

(ii) $\bmod B_{1}, \ldots, \bmod B_{m}$ are functorially finite subcategories of $\bmod A$.

(iii) All but finitely many modules in ind $A$ belong to $\bigcup_{i=1}^{m}$ ind $B_{i}$.

Recall that a full subcategory $\mathcal{X}$ of $\bmod A$ is called contravariantly finite (respectively, covariantly finite) if, for each module $M$ in $\bmod A$, the family of all homomorphisms $f$ : $X \rightarrow M$ (respectively, $g: M \rightarrow X$ ) in $\bmod A$ with $X$ in $\mathcal{X}$ is finitely generated $[4,5]$. Then $\mathcal{X}$ is called functorially finite if it is both contravariantly and covariantly finite.

The paper is organized as follows. In Section 2 we present some concepts and results on module categories of algebras, essential for further considerations. Section 3 contains known results on the module categories of cycle-finite algebras, which are applied in the proofs of our main theorems. Sections 4 and 5 are devoted to the proofs of Theorems 1.6 and 1.2 , respectively.

\section{Preliminaries}

We recall some notation, concepts and results on algebras and module categories needed in our further considerations. 
Throughout the article we will assume (without loss of generality) that algebras are basic. Recall that an algebra $A$ is basic if $A / \operatorname{rad} A$ is a product of division algebras.

Let $A$ be an algebra and $e_{1}, \ldots, e_{n}$ be a set of pairwise orthogonal primitive idempotents of $A$ such that $e_{1}+\cdots+e_{n}$ is the identity of $A$. Then $S_{i}=e_{i} A / e_{i} \operatorname{rad} A, i \in\{1, \ldots, n\}$, form a complete set of pairwise nonisomorphic simple modules in $\bmod A$. For each $i \in$ $\{1, \ldots, n\}$, let $F_{i}=\operatorname{End}_{A}\left(S_{i}\right)$ be the associated division algebra. The quiver $Q_{A}$ of $A$ is the valued quiver defined as follows:

- $\quad$ the vertices of $Q_{A}$ are the indices $1, \ldots, n$ of the chosen set $e_{1}, \ldots, e_{n}$ of primitive idempotents of $A$;

- for two vertices $i$ and $j$ in $Q_{A}$, there is an arrow $i \rightarrow j$ from $i$ to $j$ in $Q_{A}$ if and only if $e_{i}(\operatorname{rad} A) e_{j} / e_{i}(\operatorname{rad} A)^{2} e_{j} \neq 0$. Moreover, one associates to an arrow $i \rightarrow j$ in $Q_{A}$ the valuation $\left(d_{i j}, d_{i j}^{\prime}\right)$, so we have in $Q_{A}$ the valued arrow

$$
i \stackrel{\left(d_{i j}, d_{i j}^{\prime}\right)}{\longrightarrow} j,
$$

where the valuation numbers are $d_{i j}=\operatorname{dim}_{F_{j}}\left(e_{i}(\operatorname{rad} A) e_{j} / e_{i}(\operatorname{rad} A)^{2} e_{j}\right)$ and $d_{i j}^{\prime}=$ $\operatorname{dim}_{F_{i}}\left(e_{i}(\operatorname{rad} A) e_{j} / e_{i}(\operatorname{rad} A)^{2} e_{j}\right)$.

We denote by $K_{0}(A)$ the Grothendieck group of $A$. Given a module $M$ in $\bmod A$, we denote by $[M]$ its image in $K_{0}(A)$. We note that $\left[S_{1}\right], \ldots,\left[S_{n}\right]$ form a $\mathbb{Z}$-basis of $K_{0}(A)$. Hence, for two modules $M$ and $N$ in $\bmod A,[M]=[N]$ if and only if $M$ and $N$ have the same composition factors including the multiplicities. Moreover, for a module $X$ in $\bmod A,|X|$ denotes the length of $X$ over the commutative artin ring $K$.

The following fact from [59, Proposition 4.1] will be essential for our proof of Theorem 1.2.

Proposition 2.1 Let $A$ be an algebra, $M, N, X$ be modules in ind $A$, and assume that $[M]=[N]$. Then the following equalities hold:

(i) $\left|\operatorname{Hom}_{A}(X, M)\right|-\left|\operatorname{Hom}_{A}\left(M, \tau_{A} X\right)\right|=\left|\operatorname{Hom}_{A}(X, N)\right|-\left|\operatorname{Hom}_{A}\left(N, \tau_{A} X\right)\right|$.

(ii) $\left|\operatorname{Hom}_{A}(M, X)\right|-\left|\operatorname{Hom}_{A}\left(\tau_{A}^{-1} X, M\right)\right|=\left|\operatorname{Hom}_{A}(N, X)\right|-\left|\operatorname{Hom}_{A}\left(\tau_{A}^{-1} X, N\right)\right|$.

Let $A$ be an algebra. By a component of $\Gamma_{A}$ we mean a connected component of the quiver $\Gamma_{A}$. A component $\mathcal{C}$ of $\Gamma_{A}$ is called regular if $\mathcal{C}$ contains neither a projective module nor an injective module. It has been proved independently by Liu [29] and Zhang [70] that a regular component $\mathcal{C}$ of $\Gamma_{A}$ contains an oriented cycle if and only if $\mathcal{C}$ is a stable tube, that is, a component of the form $\mathbb{Z}_{\infty} /\left(\tau^{r}\right)$, for some $r \geq 1$, called the rank of $\mathcal{C}$. The $\tau_{A}$-orbit of a stable tube $\mathcal{C}$ of $\Gamma_{A}$ formed by the modules having exactly one direct predecessor is called the mouth of $\mathcal{C}$. For a module $X$ lying in a stable tube $\mathcal{C}$ of $\Gamma_{A}$, there is exactly one sectional path $X_{1} \rightarrow X_{2} \rightarrow \cdots \rightarrow X_{s}=X$ with $X_{1}$ lying on the mouth of $\mathcal{C}$, and $s$ is called the quasi-length of $X$ in $\mathcal{C}$ which we shall denote by $\mathrm{ql}(X)$. Moreover, Liu proved in [30] that a component $\mathcal{C}$ without injective modules (respectively, projective modules) contains an oriented cycle if and only if $\mathcal{C}$ is a ray tube (respectively, a coray tube), which is obtained from a stable tube by a finite number (possibly zero) ray insertions (respectively, coray insertions). A component $\mathcal{C}$ of $\Gamma_{A}$ is called almost cyclic if all but finitely many modules in $\mathcal{C}$ are cyclic (lie on oriented cycles). 
Further, a component $\mathcal{C}$ of $\Gamma_{A}$ is called coherent [35] if the following two conditions are satisfied:

(C1) For each projective module $P$ in $\mathcal{C}$ there is an infinite sectional path

$$
P=X_{1} \rightarrow X_{2} \rightarrow \cdots \rightarrow X_{i} \rightarrow X_{i+1} \rightarrow X_{i+2} \rightarrow \cdots
$$

(C2) For each injective module $I$ in $\mathcal{C}$ there is an infinite sectional path

$$
\cdots \rightarrow Y_{j+2} \rightarrow Y_{j+1} \rightarrow Y_{j} \rightarrow \cdots \rightarrow Y_{2} \rightarrow Y_{1}=I .
$$

We note that all ray tubes and coray tubes are almost cyclic and coherent. It has been proved in [35] that a component $\mathcal{C}$ of $\Gamma_{A}$ is almost cyclic and coherent if and only if $\mathcal{C}$ is a generalized multicoil, which is obtained from a finite family of stable tubes by a finite sequence of admissible operations $(\operatorname{ad} 1)-(\operatorname{ad} 5)$ and their duals $(\operatorname{ad} 1 *)-(\operatorname{ad} 5 *)$. Following [57], a subqiver $\mathcal{D}$ of $\Gamma_{A}$ is said to be generalized standard if $\operatorname{rad}_{A}^{\infty}(X, Y)=0$ for all indecomposable modules $X$ and $Y$ in $\mathcal{D}$. Finally, an indecomposable module $M$ in $\Gamma_{A}$ is said to be left stable (respectively, right stable) if $\tau_{A}^{n} M$ is nonzero for any $n>0$ (respectively, for any $n<0)$.

Let $A$ be an algebra and $\mathcal{C}=\left(\mathcal{C}_{i}\right)_{i \in I}$ a family of components of $\Gamma_{A}$. Then $\mathcal{C}$ is called sincere if every simple module in $\bmod A$ is a composition factor of a module in $\mathcal{C}$. Then $\mathcal{C}$ is said to be a separating family in $\bmod A$ if the components in $\Gamma_{A}$ split into three disjoint families, $\mathcal{P}^{A}, \mathcal{C}^{A}=\mathcal{C}$ and $\mathcal{Q}^{A}$, such that the following conditions are satisfied:

(S1) $\mathcal{C}^{A}$ is a sincere family of pairwise orthogonal generalized standard components;

(S2) $\operatorname{Hom}_{A}\left(\mathcal{Q}^{A}, \mathcal{P}^{A}\right)=0, \operatorname{Hom}_{A}\left(\mathcal{Q}^{A}, \mathcal{C}^{A}\right)=0, \operatorname{Hom}_{A}\left(\mathcal{C}^{A}, \mathcal{P}^{A}\right)=0$;

(S3) every homomorphism from $\mathcal{P}^{A}$ to $\mathcal{Q}^{A}$ in $\bmod A$ factors through the additive category $\operatorname{add}\left(\mathcal{C}^{A}\right)$ of $\mathcal{C}^{A}$.

Moreover, if $(S 1),(S 2)$ and the condition

$\left(\mathrm{S} 3^{*}\right)$ every homomorphism from $\mathcal{P}^{A}$ to $\mathcal{Q}^{A}$ in $\bmod A$ factors $\operatorname{through} \operatorname{add}\left(\mathcal{C}_{i}\right)$ for any $i \in I$

are satisfied, then $\mathcal{C}$ is said to be a strongly separating family in $\bmod A$ (see [35, 49]). We then say that $\mathcal{C}^{A}$ separates (respectively, strongly separates) $\mathcal{P}^{A}$ from $\mathcal{Q}^{A}$.

Let $A$ be an algebra. Following [35], we denote by ${ }_{c} \Gamma_{A}$ the cyclic quiver of $A$ obtained from $\Gamma_{A}$ by removing all acyclic vertices (vertices not lying on oriented cycles in $\Gamma_{A}$ ) and the arrows attached to them. Then the connected components of the translation quiver ${ }_{c} \Gamma_{A}$ are said to be cyclic components of $\Gamma_{A}$. We have the following fact proved in [35, Proposition 5.1].

Proposition 2.2 Let $A$ be an algebra and $M, N$ be modules in ind $A$. Then $M$ and $N$ belong to a cyclic component $\Gamma$ of $\Gamma_{A}$ if and only if there is an oriented cycle in $\Gamma_{A}$ containing $M$ and $N$.

Let $A$ be an algebra and $\Gamma$ be a subquiver of $\Gamma_{A}$. We denote by $\operatorname{ann}_{A}(\Gamma)$ the intersection of the annihilators $\operatorname{ann}_{A}(X)=\{a \in A \mid X a=0\}$ of all indecomposable modules $X$ in $\Gamma$. Then $\operatorname{ann}_{A}(\Gamma)$ is a two-sided ideal of $A$, and the quotient algebra $B(\Gamma)=A / \operatorname{ann}_{A}(\Gamma)$ is said to be the faithful algebra of $\Gamma$. Observe that $\Gamma$ is a subquiver of $\Gamma_{B(\Gamma)}$. 


\section{Cycle-Finite Algebras}

In this section we recall known results on the structure of module categories of cycle-finite algebras, playing a prominent role in the proofs of Theorems 1.2 and 1.6.

By a tame concealed algebra we mean a tilted algebra $C=\operatorname{\sim nd}_{H}(T)$, where $H$ is a hereditary algebra of Euclidean type $\widetilde{\mathbb{A}}_{11}, \widetilde{\mathbb{A}}_{12}, \widetilde{\mathbb{A}}_{n}, \widetilde{\mathbb{B}}_{n}, \widetilde{\mathbb{C}}_{n}, \widetilde{\mathbb{B C}}_{n}, \widetilde{B D D}_{n}, \widetilde{\mathbb{C D}}_{n}, \widetilde{\mathbb{D}}_{n}, \widetilde{\mathbb{E}}_{6}, \widetilde{\mathbb{E}}_{7}$, $\widetilde{\mathbb{E}}_{8}, \widetilde{\mathbb{F}}_{41}, \widetilde{\mathbb{F}}_{42}, \widetilde{\mathbb{G}}_{21}$, or $\widetilde{\mathbb{G}}_{22}$, and $T$ is a (multiplicity-free) tilting $H$-module from the additive category of the postprojective component of $\Gamma_{H}$. The Auslander-Reiten quiver $\Gamma_{C}$ of a tame concealed algebra $C$ is of the form

$$
\Gamma_{C}=\mathcal{P}^{C} \cup \mathcal{T}^{C} \cup \mathcal{Q}^{C},
$$

where

- $\quad \mathcal{P}^{C}$ is a postprojective component of Euclidean type containing all indecomposable projective $C$-modules;

- $\mathcal{Q}^{C}$ is a preinjective component of Euclidean type containing all indecomposable injective $C$-modules;

- $\quad \mathcal{T}^{C}$ is an infinite family of stable tubes, and all but finitely many tubes in $\mathcal{T}^{C}$ have rank one;

- $\mathcal{T}^{C}$ strongly separates $\mathcal{P}^{C}$ from $\mathcal{Q}^{C}$.

More generally, by a tilted algebra of Euclidean type we mean a tilted algebra $B=$ $\operatorname{End}_{H}(T)$, where $H$ is a hereditary algebra of Euclidean type and $T$ is a (multiplicityfree) tilting module in $\bmod H$. Assume that $B$ is a representation-infinite tilted algebra of Euclidean type. Then one of the following holds:

(1) $B$ is a domestic tubular extension of a tame concealed algebra $C$ and

$$
\Gamma_{B}=\mathcal{P}^{B} \cup \mathcal{T}^{B} \cup \mathcal{Q}^{B},
$$

where

- $\quad \mathcal{P}^{B}=\mathcal{P}^{C}$ is the postprojective component of $\Gamma_{C}$;

- $\mathcal{Q}^{B}$ is a preinjective component of Euclidean type containing all indecomposable injective $B$-modules and all indecomposable modules from the preinjective component $\mathcal{Q}^{C}$ of $\Gamma_{C}$;

- $\mathcal{T}^{B}$ is an infinite family of ray tubes, obtained from the family $\mathcal{T}^{C}$ of stable tubes of $\Gamma_{C}$ by ray insertions;

- $\mathcal{T}^{B}$ strongly separates $\mathcal{P}^{B}$ from $\mathcal{Q}^{B}$;

(2) $B$ is a domestic tubular coextension of a tame concealed algebra $C$ and

$$
\Gamma_{B}=\mathcal{P}^{B} \cup \mathcal{T}^{B} \cup \mathcal{Q}^{B},
$$

where

- $\quad \mathcal{P}^{B}$ is a postprojective component of Euclidean type containing all indecomposable projective $B$-modules and all indecomposable modules from the postprojective component $\mathcal{P}^{C}$ of $\Gamma_{C}$;

- $\mathcal{Q}^{B}=\mathcal{Q}^{C}$ is the preinjective component of $\Gamma_{C}$;

- $\mathcal{T}^{B}$ is an infinite family of coray tubes, obtained from the family $\mathcal{T}^{C}$ of stable tubes of $\Gamma_{C}$ by coray insertions;

- $\mathcal{T}^{B}$ strongly separates $\mathcal{P}^{B}$ from $\mathcal{Q}^{B}$. 
By a tubular algebra we mean a tubular extension (equivalently, tubular coextension) of a tame concealed algebra, with the Euler quadratic form positive semidefinite of corank 2 (see [28], [49], [50]). By general theory, a tubular algebra $B$ admits two different tame concealed quotient algebras $C_{0}$ and $C_{\infty}$ such that $B$ is a tubular extension of $C_{0}$ and a tubular coextension of $C_{\infty}$, and the Auslander-Reiten quiver $\Gamma_{B}$ has a disjoint union decomposition

$$
\Gamma_{B}=\mathcal{P}_{0}^{B} \cup \mathcal{T}_{0}^{B} \cup\left(\bigcup_{q \in \mathbb{Q}^{+}} \mathcal{T}_{q}^{B}\right) \cup \mathcal{T}_{\infty}^{B} \cup \mathcal{Q}_{\infty}^{B},
$$

where $\mathbb{Q}^{+}$is the set of positive rational numbers, and

- $\quad \mathcal{P}_{0}^{B}=\mathcal{P}^{C_{0}}$ is the postprojective component of $\Gamma_{C_{0}}$;

- $\mathcal{T}_{0}^{B}$ is an infinite family of ray tubes with at least one projective $B$-module, obtained from the family $\mathcal{T}^{C_{0}}$ of stable tubes of $\Gamma_{C_{0}}$ by ray insertions;

- $\mathcal{Q}_{\infty}^{B}=\mathcal{Q}^{C_{\infty}}$ is the preinjective component of $\Gamma_{C_{\infty}}$;

- $\quad \mathcal{T}_{\infty}^{B}$ is an infinite family of coray tubes with at least one injective $B$-module, obtained from the family $\mathcal{T}^{C_{\infty}}$ of stable tubes of $\Gamma_{C_{\infty}}$ by coray insertions;

- for each $q \in \mathbb{Q}^{+}, \mathcal{T}_{q}^{B}$ is an infinite family of stable tubes, containing at least one stable tube of rank bigger than one;

- for each $q \in \mathbb{Q}^{+} \cup\{0, \infty\}$, the family $\mathcal{T}_{q}^{B}$ strongly separates $\mathcal{P}_{0}^{B} \cup\left(\cup_{p<q} \mathcal{T}_{p}^{B}\right)$ from $\left(\bigcup_{p>q} \mathcal{T}_{p}^{B}\right) \cup \mathcal{Q}_{\infty}^{B}$

- $\bigcup_{q \in \mathbb{Q}^{+}} \mathcal{T}_{q}^{B}$ is the family of all sincere stable tubes of $\Gamma_{B}$ and contains all sincere indecomposable $C_{0}$-modules from the preinjective component $\mathcal{Q}^{C_{0}}$ of $\Gamma_{C_{0}}$ and all sincere indecomposable $C_{\infty}$-modules from the postprojective component $\mathcal{P}^{C_{\infty}}$ of $\Gamma_{C_{\infty}}$.

The following characterization of tame concealed algebras has been established in [60, Theorem 4.1].

Theorem 3.1 Let A be an algebra. The following statements are equivalent:

(i) $A$ is cycle-finite and $\Gamma_{A}$ admits a sincere stable tube;

(ii) A is a tame concealed algebra or a tubular algebra.

We have also the following consequence of [58, Theorem 4.1].

Theorem 3.2 Let A be a cycle-finite algebra. The following statements are equivalent:

(i) A is a tame concealed algebra;

(ii) for every nonzero idempotent e of $A$, the algebra $A / A e A$ is of finite representation type.

In particular, we obtain the following useful fact (see [58, Corolary 4.3]).

Corollary 3.3 Let A be a cycle-finite algebra. The following statements are equivalent:

(i) A is of infinite representation type;

(ii) there is an idempotent e of $A$ such that A/AeA is a tame concealed algebra.

We also mention that Theorem 3.1 describes the structure of all regular components of the Auslander-Reiten quivers of cycle-finite algebras (see [9, Proposition 2.4] or [59, Proposition 3.3] for a proof). 
Proposition 3.4 Let A be a cycle-finite algebra and $\mathcal{C}$ be a regular component of $\Gamma_{A}$. Then the following statements hold:

(i) $\mathcal{C}$ is a stable tube;

(ii) $B(\mathcal{C})$ is a tame concealed algebra or a tubular algebra.

Let $C_{1}, \ldots, C_{m}$ be a family of tame concealed algebras. Following [36], by a generalized multicoil enlargement of $C_{1}, \ldots, C_{m}$ we mean an algebra $A$ obtained by a sequence of algebra admissible operations of types (ad1)-(ad5) and their duals $(\operatorname{ad} 1 *)-(\operatorname{ad} 5 *)$ using modules from the separating families $\mathcal{T}^{C_{1}}, \ldots, \mathcal{T}^{C_{m}}$ of stable tubes $\Gamma_{C_{1}}, \ldots, \Gamma_{C_{m}}$. Then there are a unique quotient algebra $A^{(l)}$ of $A$ being a product of algebras having separating families of coray tubes (the left quasitilted algebra of $A$ ) and a unique quotient algebra $A^{(r)}$ of $A$ being a product of algebras having separating families of ray tubes (the right quasitilted algebra of $A$ ) such that $\Gamma_{A}$ has a disjoint union decomposition (see [36, Theorems C and E])

$$
\Gamma_{A}=\mathcal{P}^{A} \cup \mathcal{C}^{A} \cup \mathcal{Q}^{A},
$$

where

- $\quad \mathcal{P}^{A}$ is the left part $\mathcal{P}^{A^{(l)}}$ in a decomposition $\Gamma_{A^{(l)}}=\mathcal{P}^{A^{(l)}} \cup \mathcal{T}^{A^{(l)}} \cup \mathcal{Q}^{A^{(l)}}$ of $\Gamma_{A^{(l)}}$, with $\mathcal{T}^{A^{(l)}}$ a family of coray tubes strongly separating $\mathcal{P}^{A^{(l)}}$ from $\mathcal{Q}^{A^{(l)}}$;

- $\mathcal{Q}^{A}$ is the right part $\mathcal{Q}^{A^{(r)}}$ in a decomposition $\Gamma_{A^{(r)}}=\mathcal{P}^{A^{(r)}} \cup \mathcal{T}^{A^{(r)}} \cup \mathcal{Q}^{A^{(r)}}$ of $\Gamma_{A^{(r)}}$, with $\mathcal{T}^{A^{(r)}}$ a family of ray tubes strongly separating $\mathcal{P}^{A^{(r)}}$ from $\mathcal{Q}^{A^{(r)}}$;

- $\mathcal{C}^{A}$ is a family of generalized multicoils separating $\mathcal{P}^{A}$ from $\mathcal{Q}^{A}$, obtained from the families $\mathcal{T}^{C_{1}}, \ldots, \mathcal{T}^{C_{m}}$ of stable tubes of $\Gamma_{C_{1}}, \ldots, \Gamma_{C_{m}}$ by a sequence of translation quiver admissible operations of types (ad1)-(ad5) and their duals (ad1*)-(ad5*), corresponding to the algebra admissible operations of types (ad1)-(ad5) and their duals $(\operatorname{ad1} *)-(\operatorname{ad} 5 *)$ leading from $C_{1}, \ldots, C_{m}$ to $A$;

- $\mathcal{C}^{A}$ contains all indecomposable modules of the cyclic parts of $\mathcal{T}^{A^{(l)}}$ and $\mathcal{T}^{A^{(r)}}$;

- $\mathcal{P}^{A}$ contains all indecomposable modules of $\mathcal{P}^{A^{(r)}}$;

- $\mathcal{Q}^{A}$ contains all indecomposable modules of $\mathcal{Q}^{A^{(l)}}$.

We also mention that $\mathrm{gl} \operatorname{dim} A \leq 3$ and every module $X$ in ind $A$ satisfies $\operatorname{pd}_{A} X \leq 2$ or $\operatorname{id}_{A} X \leq 2$ (see [36, Theorem E]).

By a tame generalized multicoil algebra we mean a generalized multicoil enlargement $A$ of a finite family of tame concealed algebras such that $A^{(l)}$ and $A^{(r)}$ are products of tilted algebras of Euclidean type or tubular algebras. Moreover, a tame generalized multicoil algebra is said to be domestic if $A^{(l)}$ and $A^{(r)}$ are products of tilted algebras of Euclidean type.

The following theorem provides a characterization of tame generalized multicoil algebras (see [36, Theorems A and F]).

Theorem 3.5 Let A be an algebra. The following statements are equivalent:

(i) A is a tame generalized multicoil algebra;

(ii) $A$ is cycle-finite and $\Gamma_{A}$ admits a separating family of almost cyclic coherent components.

The following special case of [34, Theorem 1.2] will be crucial for our investigations. 
Theorem 3.6 Let A be a cycle-finite algebra and $\Gamma$ be an infinite cyclic component of $\Gamma_{A}$. Then there exist infinite full translation subquivers $\Gamma_{1}, \ldots, \Gamma_{r}$ of $\Gamma$ such that the following statements hold.

(i) For each $i \in\{1, \ldots, r\}, \Gamma_{i}$ is a cyclic coherent subquiver of $\Gamma_{A}$.

(ii) For each $i \in\{1, \ldots, r\}, B\left(\Gamma_{i}\right)$ is a tame generalized multicoil algebra.

(iii) $\Gamma_{1}, \ldots, \Gamma_{r}$ are pairwise disjoint full translation subquivers of $\Gamma$ and $\Gamma^{c c}=\Gamma_{1} \cup \ldots \cup \Gamma_{r}$ is a maximal cyclic coherent and cofinite full translation subquiver of $\Gamma$.

(iv) $B\left(\Gamma \backslash \Gamma^{c c}\right)$ is of finite representation type.

We recall also the following known facts, and their simple proofs.

Lemma 3.7 Let $A$ be an algebra, $B$ a quotient algebra, $\mathcal{T}$ a stable tube of $\Gamma_{B}$, and $\mathcal{C}$ a stable tube of $\Gamma_{A}$ containing all indecomposable modules of $\mathcal{T}$. Then $\mathcal{C}=\mathcal{T}$.

Proof Observe that there is a coray in $\mathcal{C}$ containing infinitely many modules from $\mathcal{T}$. Then for any indecomposable module $X$ in $\mathcal{C}$ there are a sequence of irreducible monomorphisms

$$
X=X_{0} \stackrel{f_{1}}{\rightarrow} X_{1} \rightarrow \cdots \rightarrow X_{r-1} \stackrel{f_{r}}{\rightarrow} X_{r}=Y
$$

and a sequence of irreducible epimorphisms

$$
Z=Y_{s} \stackrel{g_{s}}{\rightarrow} Y_{s-1} \rightarrow \cdots \rightarrow Y_{1} \stackrel{g_{1}}{\rightarrow} Y_{0}=Y
$$

in $\bmod A$ between indecomposable modules in $\mathcal{C}$ and with $Z$ from $\mathcal{T}$. Then $X$ is isomorphic to a submodule of $Y$ and $Y$ is isomorphic to a quotient module of $Z$. Therefore, $X$ is a $B$-module, because $Z$ is a $B$-module.

Lemma 3.8 Let $A$ be a cycle-finite algebra, $B$ a quotient algebra of $A$, and $\mathcal{T}$ a stable tube of $\Gamma_{B}$. Then there exists a cyclic component $\Gamma$ of $\Gamma_{A}$ containing all indecomposable modules of $\mathcal{T}$.

Proof Since $\mathcal{T}$ is a stable tube, for any modules $X$ and $Y$ in $\mathcal{T}$ there exists a cycle of irreducible homomorphisms

$$
X \stackrel{f_{1}}{\rightarrow} X_{1} \rightarrow \cdots \rightarrow X_{r-1} \stackrel{f_{r}}{\rightarrow} Y \stackrel{g_{s}}{\rightarrow} Y_{s-1} \rightarrow \cdots \rightarrow Y_{1} \stackrel{g_{1}}{\rightarrow} X
$$

in $\bmod B$ between indecomposable modules in $\mathcal{T}$. Since $A$ is cycle-finite, the homomorphisms $f_{1}, \ldots, f_{r}, g_{1}, \ldots, g_{s}$ do not belong to $\operatorname{rad}_{A}^{\infty}$, and consequently there exists an oriented cycle of irreducible homomorphisms between modules in ind $A$ containing $X$ and $Y$. Hence there is an oriented cycle in $\Gamma_{A}$ containing $X$ and $Y$. This shows that all indecomposable modules of the stable tube $\mathcal{T}$ of $\Gamma_{B}$ belong to a common cyclic component $\Gamma$ of $\Gamma_{A}$, by Proposition 2.2.

The following propositions are consequences of Theorems 3.1, 3.6, Proposition 3.4, and Lemmas 3.7, 3.8.

Proposition 3.9 Let $A$ be a cycle-finite algebra, $C$ a tame concealed quotient algebra of $A$, and $\mathcal{T}^{C}$ the family of all stable tubes of $\Gamma_{C}$. Then all but finitely many tubes of $\mathcal{T}^{C}$ are stable tubes of $\Gamma_{A}$. 
Proposition 3.10 Let $A$ be a cycle-finite algebra, $B$ a tubular quotient algebra of $A$, and

$$
\Gamma_{B}=\mathcal{P}_{0}^{B} \cup \mathcal{T}_{0}^{B} \cup\left(\bigcup_{q \in \mathbb{Q}^{+}} \mathcal{T}_{q}^{B}\right) \cup \mathcal{T}_{\infty}^{B} \cup \mathcal{Q}_{\infty}^{B}
$$

the canonical decomposition of $\Gamma_{B}$. Then all tubes in $\bigcup_{q \in \mathbb{Q}^{+}} \mathcal{T}_{q}^{B}$ are stable tubes of $\Gamma_{A}$. In particular, $\Gamma_{A}$ contains infinitely many stable tubes of rank bigger than one.

We end this section with some results towards the proof of Theorem 1.2.

The following fact has been proved in [25, 1.5] (see also [61, Proposition 3.5]).

Proposition 3.11 Let $B$ a tubular algebra. Then $\bigcap_{m \geq 1}\left(\operatorname{rad}_{B}^{\infty}\right)^{m} \neq 0$. In particular, $\operatorname{rad}_{B}^{\infty}$ is not nilpotent.

We have also the following consequence of [36, Theorems A and C].

Proposition 3.12 Let $B$ a domestic generalized multicoil algebra. Then $\left(\operatorname{rad}_{B}^{\infty}\right)^{3}=0$.

The next proposition is due to Geigle [23, Proposition 4.1].

Proposition 3.13 Let $B$ a tubular algebra. Then $K G(B)=\infty$.

We will need also the following result by Malicki [31, Theorem 1.1], extending a result proved in [69, Proposition 2.2].

Proposition 3.14 Let $B$ a domestic generalized multicoil algebra. Then $K G(B)=2$.

Finally, we present the following consequence of recent results proved in [9, Propositions 2.10 and 2.11].

Theorem 3.15 Let $A$ be a cycle-finite algebra of infinite representation type and without a tubular quotient algebra. Moreover, let $C$ be a tame concealed quotient algebra of $A$. Then the following statements hold.

(i) All but finitely many indecomposable modules from the postprojective component $\mathcal{P}^{C}$ of $\Gamma_{C}$ are directing modules.

(ii) All but finitely many indecomposable modules from the preinjective component $\mathcal{Q}^{C}$ of $\Gamma_{C}$ are directing modules in ind $A$.

\section{Proof of Theorem 1.6}

Throughout this section we assume that $A$ is a cycle-finite algebra of infinite representation type and without a tubular quotient algebra. Moreover, let $e_{1}, \ldots, e_{n}$ be a fixed set of pairwise orthogonal primitive idempotents in $A$ such that $e_{1}+\cdots+e_{n}$ is the identity $1_{A}$ of $A$. It follows from Corollary 3.3 that there is an idempotent $f_{C}$ in $A$ such that $C=A / A f_{C} A$ is a tame concealed algebra. Further, it follows from Proposition 3.9 that there is a stable tube $\mathcal{T}$ in $\Gamma_{C}$ which remains a stable tube in $\Gamma_{A}$. Moreover, $C$ is the faithful algebra $B(\mathcal{T})$ of $\mathcal{T}$, considered as a component of $\Gamma_{A}$. We note that $\mathcal{T}$ is a cyclic component of $\Gamma_{A}$. Then, applying [34, Theorem 1.2 and Propositions 2.3, 2.4], we conclude that there is a convex full 
subquiver $\Delta_{C}$ of the quiver $Q_{A}$ of $A$ such that $f_{C}=1_{A}-e_{C}$ with $e_{C}=e_{i_{1}}+\cdots+e_{i_{r}}$, for $i_{1}, \ldots, i_{r}$ being the vertices of $\Delta_{C}$. Moreover, $C$ is isomorphic to the algebra $e_{C} A e_{C}$ (see [34, Corollary 1.3]). Since the quiver $Q_{A}$ of $A$ has only finitely many convex subquivers, we conclude that $A$ admits only a finite number of tame concealed quotient algebras.

Let $C_{1}, \ldots, C_{m}$ be the family of all tame concealed quotient algebras of $A$. Fix $i \in$ $\{1, \ldots, m\}$. Let $e^{(i)}=e_{C_{i}}$ and $f^{(i)}=f_{C_{i}}$ be idempotents of $A$ such that $1_{A}=e^{(i)}+f^{(i)}$ and $C_{i}=A / A f^{(i)} A$. We identify $\bmod C_{i}$ with the full subcategory of $\bmod A$ formed by all modules $M$ in $\bmod A$ such that $M f^{(i)}=0$, or equivalently, $M=M e^{(i)}$. Moreover, let $\operatorname{res}_{i}: \bmod A \rightarrow \bmod C_{i}$ be the restriction functor which assigns to a module $X$ in $\bmod A$ the module $\operatorname{res}_{i}(X)=X e^{(i)}$ in $\bmod C_{i}$ and to a homomorphism $f: X \rightarrow Y$ in $\bmod A$ the restriction $\operatorname{res}_{i}(f): \operatorname{res}_{i}(X) \rightarrow \operatorname{res}_{i}(Y)$ of $f$ to $X e^{(i)}$. Consider the decomposition

$$
\Gamma_{C_{i}}=\mathcal{P}^{C_{i}} \cup \mathcal{T}^{C_{i}} \cup \mathcal{Q}^{C_{i}}
$$

of the Auslander-Reiten quiver $\Gamma_{C_{i}}$ of $C_{i}$, where $\mathcal{P}^{C_{i}}$ is the unique postprojective component of Euclidean type, $\mathcal{Q}^{C_{i}}$ is the unique preinjective component of Euclidean type, and $\mathcal{T}^{C_{i}}=\left(\mathcal{T}_{\lambda}^{C_{i}}\right)_{\lambda \in \Lambda_{i}}$ is an infinite family of stable tubes, strongly separating $\mathcal{P}^{C_{i}}$ from $\mathcal{Q}^{C_{i}}$. We denote by $r_{\lambda}^{(i)}$ the rank of the stable tube $\mathcal{T}_{\lambda}^{C_{i}}$, for any $\lambda \in \Lambda_{i}$. By general theory, we have $r_{\lambda}^{(i)}=1$ for all but finitely many $\lambda \in \Lambda_{i}$. Moreover, by Proposition 3.9, all but finitely many stable tubes in $\mathcal{T}^{C_{i}}$ are components of $\Gamma_{A}$. Assume now that $\mathcal{T}_{\lambda}^{C_{i}}$, for some $\lambda \in \Lambda_{i}$, is not a component of $\Gamma_{A}$. Then, applying Lemma 3.8, we conclude that there exists a cyclic component $\Gamma_{\lambda}^{(i)}$ of $\Gamma_{A}$ containing all indecomposable modules of $\mathcal{T}_{\lambda}^{C_{i}}$, and $\Gamma_{\lambda}^{(i)}$ is a full translation subquiver of a nonregular component $\mathcal{C}_{\lambda}^{(i)}$ of $\Gamma_{A}$, by Proposition 3.4 and Lemma 3.7. Hence, there is a cofinite subset $\Lambda_{i}^{\prime}$ of $\Lambda_{i}$ such that the stable tubes $\mathcal{T}_{\lambda}^{C_{i}}$, $\lambda \in \Lambda_{i}^{\prime}$, are all regular components of $\Gamma_{A}$ containing an indecomposable module from $\mathcal{T}^{C_{i}}$. We set $\Lambda_{i}^{\prime \prime}=\Lambda_{i} \backslash \Lambda_{i}^{\prime}$. We note that we may have $\Lambda_{i}=\Lambda_{i}^{\prime}$, and then $\Lambda_{i}^{\prime \prime}$ is empty. Assume $\Lambda_{i}^{\prime \prime}$ is nonempty, and take $\lambda \in \Lambda_{i}^{\prime \prime}$. Then, according to Theorem 3.6, there is a unique maximal cyclic coherent translation subquiver $\Omega_{\lambda}^{(i)}$ of the cyclic component $\Gamma_{\lambda}^{(i)}$ containing all indecomposable modules of the stable tube $\mathcal{T}_{\lambda}^{C_{i}}$ of $\Gamma_{C_{i}}$. Further, by general theory (see [35, Section 2] and [34, Proposition 3.4]), the translation quiver $\Omega_{\lambda}^{(i)}$ admits a left border $\Delta_{\lambda}^{(i)}$ and a right border $\Sigma_{\lambda}^{(i)}$ having the following properties:

- $\Delta_{\lambda}^{(i)}$ and $\Sigma_{\lambda}^{(i)}$ are finite, disjoint, and unions of sectional paths of $\Gamma_{A}$;

- $\Omega_{\lambda}^{(i)}$ is a maximal coherent cyclic subquiver of $\Gamma_{A}$ consisting of modules which are both successors of modules lying on $\Delta_{\lambda}^{(i)}$ and predecessors of modules lying on $\Sigma_{\lambda}^{(i)}$;

- $\quad$ every path in $\Gamma_{A}$ from a module in $\mathcal{C}_{\lambda}^{(i)} \backslash \Omega_{\lambda}^{(i)}$ to a module in $\Omega_{\lambda}^{(i)}$ contains a module from $\Delta_{\lambda}^{(i)}$;

- $\quad$ every path in $\Gamma_{A}$ from a module in $\Omega_{\lambda}^{(i)}$ to a module in $\mathcal{C}_{\lambda}^{(i)} \backslash \Omega_{\lambda}^{(i)}$ contains a module from $\Sigma_{\lambda}^{(i)}$.

We also mention that $\Omega_{\lambda}^{(i)}$ consists of all indecomposable modules $Z$ in $\Gamma_{\lambda}^{(i)}$ such that $\operatorname{res}_{i}(Z)$ contains an indecomposable direct summand from $\mathcal{T}_{\lambda}^{C_{i}}$, and equivalently, with $\operatorname{res}_{i}(Z)$ being an indecomposable module in $\mathcal{T}_{\lambda}^{C_{i}}$. We define

$$
\Delta^{(i)}=\bigcup_{\lambda \in \Lambda_{i}^{\prime \prime}} \Delta_{\lambda}^{(i)} \quad \text { and } \quad \Sigma^{(i)}=\bigcup_{\lambda \in \Lambda_{i}^{\prime \prime}} \Sigma_{\lambda}^{(i)} .
$$


In particular, we obtain that, if $\lambda$ and $\mu$ are different elements in $\Lambda_{i}^{\prime \prime}$, then the quivers $\Omega_{\lambda}^{(i)}$ and $\Omega_{\mu}^{(i)}$ are disjoint. We would like to stress that we may have $\Gamma_{\lambda}^{(i)}=\Gamma_{\mu}^{(i)}$ for $\lambda \neq \mu$ in $\Lambda_{i}^{\prime \prime}$. We set also $\Omega_{\lambda}^{(i)}=\mathcal{T}_{\lambda}^{C_{i}}$ for any $\lambda \in \Lambda_{i}^{\prime}$, and define

$$
\Omega^{(i)}=\bigcup_{\lambda \in \Lambda_{i}} \Omega_{\lambda}^{(i)}
$$

We observe that, for any different elements $i$ and $j$ in $\{1, \ldots, m\}$, the translation quivers $\Omega^{(i)}$ and $\Omega^{(j)}$ are disjoint. Moreover, it follows from Theorem 3.6 that all but finitely many indecomposable modules of the cyclic quiver ${ }_{c} \Gamma_{A}$ belong to the translation quiver

$$
\Omega=\bigcup_{i=1}^{m} \Omega^{(i)} .
$$

In particular, all finite cyclic components of $\Gamma_{A}$ are contained in ${ }_{c} \Gamma_{A} \backslash \Omega$ (see [34, Theorem 1.2] for the structure of such components).

Since $A$ does not admit a tubular quotient algebra, applying Proposition 3.4, we conclude that the stable tubes $\Omega_{\lambda}^{(i)}=\mathcal{T}_{\lambda}^{C_{i}}$, for $i \in\{1, \ldots, m\}$ and $\lambda \in \Lambda_{i}^{\prime}$, form the family of all regular components of $\Gamma_{A}$.

We shall discuss now the structure of left stable acyclic subquivers and right stable acyclic subquivers of $\Gamma_{A}$. Fix $i \in\{1, \ldots, m\}$. We know from Theorem 3.15 that all but finitely many indecomposable modules from the postprojective component $\mathcal{P}^{C_{i}}$ and the preinjective component $\mathcal{Q}^{C_{i}}$ of $\Gamma_{C_{i}}$ are directing modules in ind $A$. Then it follows from the proofs of [9, Propositions 3.2 and 3.4] that there exist indecomposable modules $M_{i}$ in $\mathcal{P}^{C_{i}}$ and $N_{i}$ in $\mathcal{Q}^{C_{i}}$ such that the following statements hold:

- $\quad$ the full translation subquiver $\mathcal{D}_{i}=\left(M_{i} \rightarrow\right)$ of $\Gamma_{A}$ formed by all successors of $M_{i}$ in $\Gamma_{A}$ is right stable, acyclic, and contains all but finitely many indecomposable modules of $\mathcal{P}^{C_{i}}$;

- $\quad$ the full translation subquiver $\mathcal{E}_{i}=\left(\rightarrow N_{i}\right)$ of $\Gamma_{A}$ formed by all predecessors of $N_{i}$ in $\Gamma_{A}$ is left stable, acyclic, and contains all but finitely many indecomposable modules of $\mathcal{Q}^{C_{i}}$.

We note that $\mathcal{D}_{i}$ and $\mathcal{E}_{i}$ consist entirely of directing modules, because every cycle in ind $A$ is finite. In particular, $\mathcal{D}_{i}$ and $\mathcal{E}_{i}$ have only finitely many $\tau_{A}$-orbits. In fact, there exists a hereditary algebra $H_{i}$ of Euclidean type and a tilting module $T_{i}$ in mod $H_{i}$ without indecomposable direct summands from the postprojective component $\mathcal{P}^{H_{i}}$ of $\Gamma_{H_{i}}$ such that the tilted algebra $B_{i}=\operatorname{End}_{H_{i}}\left(T_{i}\right)$ is a quotient algebra of $A$ and $\mathcal{D}_{i}$ is the image of $\mathcal{P}^{H_{i}}$ via the functor $F_{i}=\operatorname{Ext}_{H_{i}}^{1}\left(T_{i},-\right): \bmod H_{i} \rightarrow \bmod B_{i}$ (see the dual of [33, Theorem 2.2]). In particular, the images of the indecomposable projective modules in $\mathcal{P}^{H_{i}}$ via $F_{i}$ form a Euclidean section $\Phi_{i}$ in $\mathcal{D}_{i}$ having the module $M_{i}$ as a unique source. Similarly, there exists a hereditary algebra $H_{i}^{*}$ of Euclidean type and a tilting module $T_{i}^{*}$ in $\bmod H_{i}^{*}$ without indecomposable direct summands from the preinjective component $\mathcal{Q}^{H_{i}^{*}}$ of $\Gamma_{H_{i}^{*}}$ such that the tilted algebra $B_{i}^{*}=\operatorname{End}_{H_{i}^{*}}\left(T_{i}^{*}\right)$ is a quotient algebra of $A$ and $\mathcal{E}_{i}$ is the image of $\mathcal{Q}^{H_{i}^{*}}$ via the functor $F_{i}^{*}=\operatorname{Hom}_{A}\left(T_{i}^{*},-\right): \bmod H_{i}^{*} \rightarrow \bmod B_{i}^{*}(\operatorname{see}[33$, Theorem 2.2]). Moreover, the images of the indecomposable injective modules in $\mathcal{Q}^{H_{i}^{*}}$ via $F_{i}^{*}$ form a Euclidean section $\Psi_{i}$ in $\mathcal{E}_{i}$ having the module $N_{i}$ as a unique sink. By general theory, $B_{i}$ is a tubular coextension of $C_{i}$ and $B_{i}^{*}$ is a tubular extension of $C_{i}$, and hence there are at most finitely many isomorphism classes of modules $Z$ in ind $B_{i}$ (respectively, in ind $B_{i}^{*}$ ) with $\operatorname{res}_{i}(Z)=0$. Therefore, we may assume that $\operatorname{res}_{i}(Z) \neq 0$ for all modules $X$ in $\mathcal{D}_{i}$ and $\operatorname{res}_{i}(Y) \neq 0$ for all modules $Y$ in 
$\mathcal{E}_{i}$. It follows from [33, Theorem 2] and its dual that all but finitely many acyclic modules of $\Gamma_{A}$ (equivalently, directing modules in ind $A$ ) belong to the union of translation quivers

$$
\mathcal{D}_{1} \cup \cdots \cup \mathcal{D}_{m} \cup \mathcal{E}_{1} \cup \cdots \cup \mathcal{E}_{m} .
$$

Fix $i \in\{1, \ldots, m\}$. Let $X$ be a module in ind $A$ which does not belong to $\Omega^{(i)}$. We claim that $\operatorname{res}_{i}(X)$ belongs to the additive category $\operatorname{add}\left(\mathcal{P}^{C_{i}} \cup \mathcal{Q}^{C_{i}}\right)$. Assume to the contrary that $\operatorname{res}_{i}(X)$ is nonzero and admits an indecomposable direct summand $U$ from $\mathcal{T}_{\lambda}^{C_{i}}$, for some $\lambda \in \Lambda_{i}$. Observe that then $\operatorname{Hom}_{A}(U, X)=\operatorname{Hom}_{C_{i}}\left(U, \operatorname{res}_{i}(X)\right) \neq 0$. Since $C_{i}$ is a quotient algebra of $A$, we may consider the largest right $C_{i}$-submodule $Y$ of $X$. Clearly, then $\operatorname{Hom}_{A}(U, X)=\operatorname{Hom}_{C_{i}}(U, Y)$, and hence $Y$ is nonzero. Let $Y=V \oplus W$ be a decomposition of $Y$ in $\bmod C_{i}$ such that $W$ is a maximal direct summand of $Y$ with all indecomposable direct summands from the family $\mathcal{T}^{C_{i}}$. Recall that $\Gamma_{C_{i}}=\mathcal{P}^{C_{i}} \cup \mathcal{T}^{C_{i}} \cup \mathcal{Q}^{C_{i}}$, where $\mathcal{T}^{C_{i}}=\left(\mathcal{T}_{\varrho}^{C_{i}}\right)_{\varrho \in \Lambda_{i}}$ strongly separates $\mathcal{P}^{C_{i}}$ from $\mathcal{Q}^{C_{i}}$. Since $\Lambda_{i}^{\prime}$ is infinite, we may choose an element $\mu \in \Lambda_{i}^{\prime} \backslash\{\lambda\}$ such that $\operatorname{Hom}_{C_{i}}(R, W)=0$ for any indecomposable module $R$ in $\mathcal{T}_{\mu}^{C_{i}}$. By general theory, the stable tubes $\mathcal{T}_{\lambda}^{C_{i}}$ and $\mathcal{T}_{\mu}^{C_{i}}$ have common composition factors, that is, there exist indecomposable modules $M$ in $\mathcal{T}_{\lambda}^{C_{i}}$ and $N$ in $\mathcal{T}_{\mu}^{C_{i}}$ with $[M]=[N]$. We note that then $r_{\lambda}^{(i)}$ divides $\mathrm{ql}(M)$ and $r_{\mu}^{(i)} \operatorname{divides} \mathrm{ql}(N)$ (see [59, Corollary 4.6]). Moreover, we may choose $M$ and $N$ with $[M]=[N]$ such that $\operatorname{Hom}_{C_{i}}(M, U) \neq 0$, and consequently $\operatorname{Hom}_{A}(M, X) \neq 0$ (see [59, Theorem 4.3 and Corollary 4.6]). Let $L$ be an indecomposable module in $\mathcal{Q}^{C_{i}}$. Then $\tau_{C_{i}}^{-1} L$ is either 0 or an indecomposable module in $\mathcal{Q}^{C_{i}}$, and hence $\operatorname{Hom}_{C_{i}}\left(\tau_{C_{i}}^{-1} L, M\right)=0$ and $\operatorname{Hom}_{C_{i}}\left(\tau_{C_{i}}^{-1} L, N\right)=0$. Then it follows from Proposition 2.1 that $\left|\operatorname{Hom}_{C_{i}}(M, L)\right|=\left|\operatorname{Hom}_{C_{i}}(N, L)\right|$. Clearly, we have $\operatorname{Hom}_{C_{i}}(M, T)=0$ and $\operatorname{Hom}_{C_{i}}(N, T)=0$ for any indecomposable module $T$ in $\mathcal{P}^{C_{i}}$. Therefore, we obtain that $\left|\operatorname{Hom}_{C_{i}}(M, V)\right|=\left|\operatorname{Hom}_{C_{i}}(N, V)\right|$. Observe that then $\operatorname{Hom}_{A}\left(\tau_{A}^{-1} X, M\right)=0$. Indeed, if $\operatorname{Hom}_{A}\left(\tau_{A}^{-1} X, M\right) \neq 0$, then we have in ind $A$ a cycle of the form

$$
M \rightarrow X \rightarrow E \rightarrow \tau_{A}^{-1} X \rightarrow M
$$

and hence a contradiction, because $M$ belongs to $\Omega_{\lambda}^{(i)}, X$ does not belong to $\Omega_{\lambda}^{(i)}$, and $A$ is cycle-finite. Applying Proposition 2.1 again, we obtain the equality

$$
\left|\operatorname{Hom}_{A}(M, X)\right|=\left|\operatorname{Hom}_{A}(N, X)\right|-\left|\operatorname{Hom}_{A}\left(\tau_{A}^{-1} X, N\right)\right|,
$$

and hence $\operatorname{Hom}_{A}(N, X) \neq 0$. We note that $X$ does not belong to $\mathcal{T}_{\mu}^{C_{i}}=\Omega_{\mu}^{(i)}$, because $\operatorname{Hom}_{A}(M, X) \neq 0$ and the stable tubes $\mathcal{T}_{\lambda}^{C_{i}}$ and $\mathcal{T}_{\mu}^{C_{i}}$ are orthogonal. Then we infer as above that $\operatorname{Hom}_{A}\left(\tau_{A}^{-1} X, N\right)=0$. Therefore, we have $\left|\operatorname{Hom}_{A}(M, X)\right|=\left|\operatorname{Hom}_{A}(N, X)\right|$. We have also the equalities

$$
\begin{aligned}
\left|\operatorname{Hom}_{A}(M, X)\right| & =\left|\operatorname{Hom}_{C_{i}}(M, Y)\right|=\left|\operatorname{Hom}_{C_{i}}(M, V)\right|+\left|\operatorname{Hom}_{C_{i}}(M, W)\right|, \\
\left|\operatorname{Hom}_{A}(N, X)\right| & =\left|\operatorname{Hom}_{C_{i}}(N, Y)\right|=\left|\operatorname{Hom}_{C_{i}}(N, V)\right|+\left|\operatorname{Hom}_{C_{i}}(N, W)\right|,
\end{aligned}
$$

and hence $\left|\operatorname{Hom}_{C_{i}}(M, W)\right|=\left|\operatorname{Hom}_{C_{i}}(N, W)\right|=0$, by the choice of $\mu$. This is a contradiction because $U$ is a direct summand of $W$ and $\operatorname{Hom}_{A}(M, U) \neq 0$. Therefore, indeed $\operatorname{res}_{i}(X)$ is a module in $\operatorname{add}\left(\mathcal{P}^{C_{i}} \cup \mathcal{Q}^{C_{i}}\right)$.

Consider now the faithful algebra $B(\Omega)$ of the cyclic quiver $\Omega$. Then it follows from Theorem 3.6, the theory of generalized multicoil algebras [36], and the assumption imposed on $A$, that $B(\Omega)$ is a tame generalized multicoil enlargement (not necessarily indecomposable) 
of the family $C_{1}, \ldots, C_{m}$ of all tame concealed quotient algebras of $A$ such that the following statements hold:

- the left quasitilted algebra $B(\Omega)^{(l)}$ of $B(\Omega)$ is a product $B_{1}^{(l)} \times \cdots \times B_{m}^{(l)}$ of tilted algebras $B_{1}^{(l)}, \ldots, B_{m}^{(l)}$ of Euclidean type such that, for any $i \in\{1, \ldots, m\}$, the translation quiver $\mathcal{D}_{i}=F_{i}\left(\mathcal{P}^{H_{i}}\right)$ is a cofinite full translation subquiver of the postprojective component $\mathcal{P}^{B_{i}^{(l)}}$ of $\Gamma_{B_{i}^{(l)}}$, which is closed under successors in $\Gamma_{A}$;

- the right quasitilted algebra $B(\Omega)^{(r)}$ of $B(\Omega)$ is a product $B_{1}^{(r)} \times \cdots \times B_{m}^{(r)}$ of tilted algebras $B_{1}^{(r)}, \ldots, B_{m}^{(r)}$ of Euclidean type such that, for any $i \in\{1, \ldots, m\}$, the translation quiver $\mathcal{E}_{i}=F_{i}^{*}\left(\mathcal{Q}^{H_{i}^{*}}\right)$ is a cofinite full translation subquiver of the preinjective component $\mathcal{Q}^{B_{i}^{(r)}}$ of $\Gamma_{B_{i}^{(r)}}$, which is closed under predecessors in $\Gamma_{A}$;

- $\Omega$ is the cyclic part of the family $\mathcal{C}^{B(\Omega)}$ of generalized multicoils of $\Gamma_{B(\Omega)}$, separating $\mathcal{P}^{B(\Omega)}=\mathcal{P}^{B(\Omega)^{(l)}}=\mathcal{P}^{B_{1}^{(l)}} \cup \cdots \cup \mathcal{P}^{B_{m}^{(l)}}$ from $\mathcal{Q}^{B(\Omega)}=\mathcal{Q}^{B(\Omega)^{(r)}}=\mathcal{Q}^{B_{1}^{(r)}} \cup \cdots \cup \mathcal{Q}^{B_{m}^{(r)}}$.

Moreover, it follows from the structure of generalized multicoils [35, 36] that, for any $i \in$ $\{1, \ldots, m\}$, there is a quotient algebra $B_{i}$ of $B(\Omega)$ such that the following statements hold:

- $\quad B_{i}$ is a generalized multicoil enlargement of the tame concealed algebra $C_{i}$;

- $B_{i}^{(l)}$ is the left quasitilted algebra of $B_{i}$;

- $B_{i}^{(r)}$ is the right quasitilted algebra of $B_{i}$;

- $\Omega^{(i)}=\bigcup_{\lambda \in \Lambda_{i}} \Omega_{\lambda}^{(i)}$ is the family of all maximal cyclic coherent translation subquivers of the Auslander-Reiten quiver $\Gamma_{B_{i}}$ of $B_{i}$.

In particular, we obtain that the translation quiver

$$
\mathcal{B}_{i}=\mathcal{D}_{i} \cup \Omega^{(i)} \cup \mathcal{E}_{i}
$$

is a cofinite full translation subquiver of $\Gamma_{B_{i}}$.

For each $i \in\{1, \ldots, m\}$, we denote by $\mathcal{X}_{i}$ the full additive subcategory of $\bmod A$ generated by all indecomposable modules in $\mathcal{B}_{i}$. Moreover, denote by $\mathcal{X}_{0}$ the full additive subcategory of $\bmod A$ generated by the family $\mathcal{B}_{0}$ of all indecomposable modules in $\bmod A$ which are not in $\mathcal{B}_{1}, \ldots, \mathcal{B}_{m}$. Observe that $\mathcal{B}_{0}$ contains only finitely many indecomposable modules. In particular, we obtain that $\mathcal{X}_{0}$ is a functorially finite subcategory of $\bmod A$. We will prove now that $\mathcal{X}_{1}, \ldots, \mathcal{X}_{m}$ are functorially finite subcategories of $\bmod A$.

Fix $i \in\{1, \ldots, m\}$. We denote by $R_{i}$ the direct sum of all indecomposable modules in $\Omega^{(i)}$ lying on the union $\Sigma^{(i)}$ of the right borders $\Sigma_{\lambda}^{(i)}$ of the maximal cyclic coherent translation quivers $\Omega_{\lambda}^{(i)}, \lambda \in \Lambda_{i}^{\prime \prime}$, if $\Lambda_{i}^{\prime \prime}$ is not empty, and $R_{i}=0$ otherwise. Moreover, we denote by $V_{i}$ the direct sum of all indecomposable modules in $\mathcal{E}_{i}$ lying on the Euclidean subquiver $\Psi_{i}$. Dually, we denote by $L_{i}$ the direct sum of all indecomposable modules in $\Omega^{(i)}$ lying on the union $\Delta^{(i)}$ of the left borders $\Delta_{\lambda}^{(i)}$ of the maximal cyclic coherent translation quivers $\Omega_{\lambda}^{(i)}, \lambda \in \Lambda_{i}^{\prime \prime}$, if $\Lambda_{i}^{\prime \prime}$ is not empty, and $L_{i}=0$ otherwise. Finally, we denote by $U_{i}$ the direct sum of all indecomposable modules in $\mathcal{D}_{i}$ lying on the Euclidean subquiver $\Phi_{i}$.

Let $X$ be a module in ind $A$ which does not belong to $\mathcal{X}_{i}$. Moreover, let $Y$ be an indecomposable module in $\mathcal{X}_{i}$ and $g: Y \rightarrow X$ a nonzero homomorphism in $\bmod A$. We will prove that $g$ factors through a module of the form $\left(R_{i} \oplus V_{i}\right)^{s}$, for some positive integer $s$. Let $Z$ be the image of $g$. Consider a decomposition $Z=Z_{1} \oplus Z_{2}$, where $Z_{1}$ is a maximal direct summand of $Z$ with $\operatorname{res}_{i}\left(Z_{1}\right)=0$, and the homomorphisms $g_{1}: Y \rightarrow Z_{1}$ and $g_{2}: Y \rightarrow Z_{2}$ induced by $g$. It follows from the previous considerations that $\operatorname{res}_{i}(X)$ is 
a module from $\operatorname{add}\left(\mathcal{P}^{C_{i}} \cup \mathcal{Q}^{C_{i}}\right)$, because $X$ does not belong to $\Omega^{(i)}$. Assume first that $Y$ belongs to $\mathcal{D}_{i}$. Then $Z$ is a right $B_{i}^{(l)}$-submodule of $X$. Moreover, there is a positive integer $a$ such that, for any integer $b \geq a, g$ factors through a direct sum of modules lying on the Euclidean subquiver $\tau_{A}^{-b} \Phi_{i}$ of $\mathcal{D}_{i}$. In particular, we conclude that $\operatorname{res}_{i}\left(Z_{2}\right)$ is a direct sum of indecomposable modules from $\mathcal{Q}^{C_{i}}$. Then we conclude that $g_{1}$ factors through a module $R_{i}^{k}$ and $g_{2}$ factors through a module $V_{i}^{t}$ for some positive integers $k$ and $t$. Assume now that $Y$ belongs to $\Omega^{(i)}$. Then $\operatorname{res}_{i}\left(Z_{2}\right)$ is a direct sum of modules lying in $\mathcal{Q}^{C_{i}}$, because $\operatorname{Hom}_{B_{i}}\left(\Omega^{(i)}, \mathcal{P}^{B_{i}^{(l)}}\right)=0$, and hence $Z_{2}$ is a right $B_{i}^{(r)}$-module from the additive category of $\mathcal{Q}^{B_{i}^{(r)}}$. Thus we conclude that $g_{2}$ factors through a module $V_{i}^{t}$ for some positive integer $t$. Clearly, we have $\operatorname{res}_{i}\left(g_{1}\right)=0$. Assume $g_{1} \neq 0$. Then $Y$ belongs to $\Omega_{\lambda}^{(i)}$ for some $\lambda \in \Lambda_{i}^{\prime \prime}$. But then $g_{1}$ factors through a module of the form $R_{i}^{k} \oplus V_{i}^{t}$ for some positive integers $k$ and $t$. Finally, assume that $Y$ belongs to $\mathcal{E}_{i}$. Obviously then $g$ factors through a module $V_{i}^{t}$ for some positive integer $t$. Summing up, we conclude that $g$ factors through a module of the form $\left(R_{i} \oplus V_{i}\right)^{s}$ for some positive integer $s$. Moreover, $\operatorname{Hom}_{A}\left(R_{i} \oplus V_{i}, X\right)$ is a finitely generated module over the commutative artin ring $K$. Then we conclude that the restriction $\left.\operatorname{Hom}_{A}(-, X)\right|_{\mathcal{X}_{i}}$ of the contravariant functor $\operatorname{Hom}_{A}(-, X):(\bmod A)^{\mathrm{op}} \rightarrow \mathcal{A} b$ to $\mathcal{X}_{i}$ is a finitely generated functor, being an epimorphic image of a contravariant functor $\operatorname{Hom}_{A}\left(-,\left(R_{i} \oplus V_{i}\right)^{p}\right): \mathcal{X}_{i}^{\text {op }} \rightarrow \mathcal{A} b$, for some positive integer $p$. Therefore, $\mathcal{X}_{i}$ is a contravariantly finite subcategory of $\bmod A$. Dually, we conclude that every nonzero homomorphism $f: X \rightarrow Y$ in $\bmod A$ with $Y$ an indecomposable module in $\mathcal{X}_{i}$ factors through a module of the form $\left(L_{i} \oplus U_{i}\right)^{t}$, for some positive integer $t$. Moreover, $\operatorname{Hom}_{A}\left(X, L_{i} \oplus U_{i}\right)$ is a finitely generated module over the commutative artin ring $K$. Then we conclude that the restriction $\operatorname{Hom}_{A}(X,-) \mid \mathcal{X}_{i}$ of the covariant functor $\operatorname{Hom}_{A}(X,-): \bmod A \rightarrow \mathcal{A} b$ to $\mathcal{X}_{i}$ is a finitely generated functor, being an epimorphic image of a covariant functor $\operatorname{Hom}_{A}\left(\left(L_{i} \oplus U_{i}\right)^{q},-\right): \mathcal{X}_{i} \rightarrow \mathcal{A} b$, for some positive integer $q$. Hence, $\mathcal{X}_{i}$ is a covariantly finite subcategory of $\bmod A$. Therefore, we proved that $\mathcal{X}_{i}$ is a functorially finite subcategory of $\bmod A$. Finally, since $\mathcal{X}_{i}$ is a full subcategory of $\bmod B_{i}$ containing all but finitely many modules of ind $B_{i}$, we obtain that $\bmod B_{i}$ is also a functorially finite subcategory of $\bmod A$.

Summing up, we conclude that $\bmod B_{1}, \ldots, \bmod B_{n}$ are functorially finite subcategories of $\bmod A$ and all but finitely many modules in ind $A$ belong to $\bigcup_{i=1}^{m}$ ind $B_{i}$. This finishes the proof of Theorem 1.6.

\section{Proof of Theorem 1.2}

The implications (v) $\Rightarrow$ (vi), (iii) $\Rightarrow$ (vi), (i) $\Rightarrow$ (vi) follow from Propositions 3.10, 3.11, 3.13 , respectively. The implications (ii) $\Rightarrow$ (i) and (iv) $\Rightarrow$ (iii) are obvious. Hence, it remains to prove that (vi) implies (ii), (iv), (v).

Let $A$ be a cycle-finite algebra of infinite representation type and without a tubular quotient algebra. Let $C_{1}, \ldots, C_{m}$ be the family of all tame concealed quotient algebras of $A$. We keep the notation introduced in Section 4.

It follows from Proposition 3.4, and the assumption imposed on $A$, that the stable tubes $\Omega_{\lambda}^{(i)}=\mathcal{T}_{\lambda}^{C_{i}}$, for $i \in\{1, \ldots, m\}$ and $\lambda \in \Lambda_{i}^{\prime}$, form the family of all regular components of $\Gamma_{A}$. Moreover, all but finitely many stable tubes in this family have rank one. Since the number of nonregular components of $\Gamma_{A}$ is finite, we conclude that all but finitely many components of $\Gamma_{A}$ are stable tubes of rank one. Hence, (vi) implies (v). 
We know from Section 4 that there exist domestic generalized multicoil enlargements $B_{1}, \ldots, B_{m}$ of $C_{1}, \ldots, C_{m}$, respectively, satisfying the the following properties:

- for each $i \in\{1, \ldots, m\}$, the translation quiver $\mathcal{B}_{i}=\mathcal{D}_{i} \cup \Omega^{(i)} \cup \mathcal{E}_{i}$ is a cofinite full translation subquiver of $\Gamma_{B_{i}}$;

- the family $\mathcal{B}_{0}$ of all modules in ind $A$ which do not belong to $\mathcal{B}_{1} \cup \cdots \cup \mathcal{B}_{m}$ is finite;

- for each $i \in\{0,1, \ldots, m\}$, the additive category $\mathcal{X}_{i}$ of $\bmod A$ generated by the indecomposable modules in $\mathcal{B}_{i}$ is contravariantly finite.

We will show now that $K G(A)=2$, and hence (vi) implies (ii). Observe first that the projective cover of any functor in $\mathcal{F}(A)$ is a functor $\operatorname{Hom}_{A}(-, M)$ for a module $M$ in $\bmod A$. Hence it is enough to consider contravariant Hom-functors. It follows from Proposition 3.14 that $K G\left(B_{i}\right)=2$, and hence $K G\left(\mathcal{X}_{i}\right)=2$ for any $i \in\{1, \ldots, m\}$. Clearly, we have $K G\left(\mathcal{X}_{0}\right)=0$. Take now an indecomposable module $X$ in ind $A$ and the associated contravariant functor $\operatorname{Hom}_{A}(-, X)$ from $\bmod A$ to $\mathcal{A} b$. Since $\mathcal{X}_{0}, \mathcal{X}_{1}, \ldots, \mathcal{X}_{m}$ are contravariantly finite subcategories of $\bmod A$, generating the whole category $\bmod A$, we conclude that $F$ becomes of finite length in the quotient category $\mathcal{F}(A) / \mathcal{F}(A)_{1}$. This shows that $K G(A) \leq 2$. On the other hand, we have $2=K G\left(\mathcal{B}_{i}\right) \leq K G(A)$, for any $i \in\{1, \ldots, m\}$. Therefore, indeed $K G(A)=2$.

Finally, we prove that $\operatorname{rad}_{A}^{\infty}$ is nilpotent, and hence (vi) implies (iv). Let $W_{0}$ be the direct sum of all indecomposable modules in $\mathcal{X}_{0}$. Then $\operatorname{End}_{A}\left(W_{0}\right)$ is an artin algebra, and hence the radical of $\operatorname{End}_{A}\left(W_{0}\right)$ is nilpotent, say $\left(\operatorname{radEnd}_{A}\left(W_{0}\right)\right)^{l}=0$ for some positive integer $l$. Let $n$ be a natural number such that $n \geq l+4 m$. We claim that $\left(\operatorname{rad}_{A}^{\infty}\right)^{n}=0$. Suppose it is not the case. Then there exists a sequence

$$
Z_{0} \stackrel{f_{1}}{\rightarrow} Z_{1} \stackrel{f_{2}}{\rightarrow} Z_{2} \rightarrow \cdots \rightarrow Z_{n-1} \stackrel{f_{n}}{\rightarrow} Z_{n}
$$

of homomorphisms in $\operatorname{rad}_{A}^{\infty}$ between modules in ind $A$ such that $f_{n} \ldots f_{2} f_{1} \neq 0$. It follows from the choice of $n$ that there are $p<q<r<s$ in $\{0,1, \ldots, n\}$ such that $Z_{p}, Z_{q}, Z_{r}, Z_{s}$ belong to $\mathcal{X}_{i}$, for some $i \in\{1, \ldots, m\}$. Let $u=f_{q} \ldots f_{p+1}: Z_{p} \rightarrow Z_{q}$, $v=f_{r} \ldots f_{q+1}: Z_{q} \rightarrow Z_{r}, w=f_{s} \ldots f_{r+1}: Z_{r} \rightarrow Z_{s}$. Since $\mathcal{X}_{i}$ is a subcategory of $\bmod B_{i}$, the homomorphisms $u, v, w$ belong to $\operatorname{rad}_{B_{i}}^{\infty}$, and hence $w v u$ is a nonzero homomorphism in $\left(\operatorname{rad}_{B_{i}}^{\infty}\right)^{3}$. This contradicts Proposition 3.12. Therefore, $\operatorname{rad}_{A}^{\infty}$ is nilpotent.

Acknowledgments This work was supported by the research grant DEC-2011/02/A/ST1/00216 of the National Science Center Poland.

Open Access This article is distributed under the terms of the Creative Commons Attribution 4.0 International License (http://creativecommons.org/licenses/by/4.0/), which permits unrestricted use, distribution, and reproduction in any medium, provided you give appropriate credit to the original author(s) and the source, provide a link to the Creative Commons license, and indicate if changes were made.

\section{References}

1. Assem, I., Skowroński, A.: Algebras with cycle-finite derived categories. Math. Ann. 280, 441-463 (1988)

2. Assem, I., Skowroński, A.: Minimal representation-infinite coil algebras. Manuscripta Math. 67, 305331 (1990)

3. Auslander, M.: Representation theory of artin algebras II. Comm. Algebra. 1, 269-310 (1974)

4. Auslander, M.: A functorial approach to representation theory. In: Representations of Algebras, Lecture Notes in Math., vol. 944, pp. 105-179. Springer, Berlin (1982) 
5. Auslander, M., Reiten, I.: Applications of contravariantly finite subcategories. Adv. Math 86, 111-152 (1991)

6. Auslander, M., Reiten, I., Smalø, S.O.: Representation Theory of Artin Algebras. In: Cambridge Studies in Advanced Mathematics, vol. 36. Cambridge University Press, Cambridge (1995)

7. Baer, D.: Wild hereditary artin algebras and linear methods. Manuscripta Math. 55, 69-82 (1986)

8. Bautista, R.: On algebras of strongly unbounded representation type. Comment. Math. Helv. 60, 392 399 (1985)

9. Białkowski, J., Skowroński, A.: Cycles of modules and finite representation type. Preprint (2015)

10. Białkowski, J., Skowroński, A., Skowyrski, A., Wiśniewski, P.: Cycle-finite algebras of semiregular type. Colloq. Math. 129, 211-247 (2012)

11. Bongartz, K.: Indecomposables are standard. Comment. Math. Helv. 60, 400-410 (1985)

12. Burke, K., Prest, M.: The Ziegler and Zariski spectra of some domestic string algebras. Algebr. Represent. Theory 5, 211-234 (2002)

13. Coelho, F.U., Marcos, E.N., Merklen, H.A., Skowroński, A.: Module categories with infinite radical square zero are of finite type. Comm. Algebra 22, 4511-4517 (1994)

14. Crawley-Boevey, W.W.: On tame algebras and bocses. Proc. London Math. Soc. 56, 451-483 (1988)

15. Crawley-Boevey, W.: Tame algebras and generic modules. Proc. London Math. Soc. 63, 241-265 (1991)

16. Dowbor, P., Skowroński, A.: Galois coverings of representation-infinite algebras. Comment. Math. Helv 62, 311-337 (1987)

17. Drozd, J.A.: Tame and wild matrix problems. In: Representation Theory II, Lecture Notes in Math., vol. 832, pp. 242-258. Springer, Berlin (1980)

18. Faith, C.: Algebra: Rings, Modules and Categories I. Springer, Berlin (1973)

19. Farnsteiner, R., Skowroński, A.: Classification of restricted Lie algebras with tame principal block. J. Reine Angew. Math. 546, 1-45 (2002)

20. Farnsteiner, R., Skowroński, A.: Galois actions and blocks of tame infinitesimal group schemes. Trans. Amer. Math. Soc. 359, 5867-5898 (2007)

21. Gabriel, P.: Des catégories abéliennes. Bull. Soc. Math. France 90, 323-448 (1962)

22. Geigle, W.: The Krull-Gabriel dimension of the representation theory of a tame hereditary artin algebra and applications to the structure of exact sequences. Manuscripta Math. 54, 83-106 (1985)

23. Geigle, W.: Krull dimension and artin algebras. In: Representation Theory I, Lecture Notes in Math., vol. 1177, pp. 135-155. Springer, Berlin (1986)

24. Kerner, O.: Tilting wild algebras. J. London Math. Soc. 39, 29-47 (1989)

25. Kerner, O., Skowroński, A.: On module categories with nilpotent infinite radical. Compositio Math. 77, 313-333 (1991)

26. Krause, H.: Generic modules over artin algebras. Proc. London Math. Soc. 76, 276-306 (1998)

27. Krause, H.: The spectrum of a module category. Mem. Amer. Math. Soc. 149(707), No. 707 (2001)

28. Lenzing, H., de la Peña, J.A.: Concealed-canonical algebras and separating tubular families. Proc. London Math. Soc. 78, 513-540 (1999)

29. Liu, S.: Degrees of irreducible maps and the shapes of Auslander-Reiten quivers. J. London Math. Soc. 45, 32-54 (1992)

30. Liu, S.: Semi-stable components of an Auslander-Reiten quiver. J. London Math. Soc. 47, 405-416 (1993)

31. Malicki, P.: Krull dimension of tame generalized multicoil algebras. Algebr. Represent. Theory 18, 881894 (2015)

32. Malicki, P., de la Peña, J.A., Skowroński, A.: Cycle-finite module categories. In: Algebras, Quivers and Representations, Abel Symposium, vol. 8, pp. 209-252. Springer, Heidelberg (2013)

33. Malicki, P., de la Peña, J.A., Skowroński, A.: On the number of terms in the middle of almost split sequences over cycle-finite artin algebras. Cent. Eur. J. Math. 12, 39-45 (2014)

34. Malicki, P., de la Peña, J.A., Skowroński, A.: Finite cycles of indecomposable modules. J. Pure Appl. Algebra 219, 1761-1799 (2015)

35. Malicki, P., Skowroński, A.: Almost cyclic coherent components of an Auslander-Reiten quiver. J. Algebra 229, 695-749 (2000)

36. Malicki, P., Skowroński, A.: Algebras with separating almost cyclic coherent Auslander-Reiten components. J. Algebra 291, 208-237 (2005)

37. Malicki, P., Skowroński, A.: Cycle-finite algebras with finitely many $\tau$-rigid indecomposable modules. Comm. Algebra. In press (2015)

38. de la Peña, J.A., Skowroński, A.: Algebras with cycle-finite Galois coverings. Trans. Amer. Math. Soc. 363, 4309-4336 (2011) 
39. Peng, L.G., Xiao, J.: On the number of $D$ Tr-orbits containing directing modules. Proc. Amer. Math. Soc. 118, 753-756 (1993)

40. Popescu, N.: Abelian Categories with Applications to Rings and Modules. Academic, London (1973). London Mathematical Society Monographs, No. 3

41. Prest, M.: Model Theory and Modules. London Mathematical Society Lecture Note Series, vol. 130. Cambridge University Press, Cambridge (1988)

42. Prest, M.: Topological and geometric aspects of the Ziegler spectrum. In: Infinite Length Modules, Trends Math., pp. 369-392. Birkhäuser, Basel (2000)

43. Prest, M.: Purity, Spectra and Localization. Encyclopedia Mathematics and Applications, vol. 121. Cambridge University Press, Cambridge (2009)

44. Prest, M., Puninski, G.: Krull-Gabriel dimension of 1-domestic string algebras. Algebr. Represent. Theory 9, 337-358 (2006)

45. Puninski, G.: Krull-Gabriel dimension and Cantor-Bendixson rank of 1-domestic string algebras. Colloq. Math. 127, 185-211 (2012)

46. Reiten, I., Skowroński, A.: Characterizations of algebras with small homological dimensions. Adv. Math. 179, 122-154 (2003)

47. Reiten, I., Skowroński, A.: Generalized double tilted algebras. J. Math. Soc. Japan 56, 269-288 (2004)

48. Ringel, C.M.: Tame algebras. In: Representation Theory I, Lecture Notes in Math., vol. 831, pp. 137287. Springer, Berlin (1980)

49. Ringel, C.M.: Tame Algebras and Integral Quadratic Forms, Lecture Notes in Mathematics, vol. 1099. Springer, Berlin (1984)

50. Ringel, C.M.: The canonical algebras. With an appendix by William Crawley-Boevey. In: Topics in Algebra, Part 1, Banach Center Publ., vol. 26, pp. 407-432. PWN, Warsaw (1990)

51. Schröer, J.: On the infinite radical of a module category. Proc. London Math. Soc. 81, 651-674 (2000)

52. Schröer, J.: On the Krull-Gabriel dimension of an algebra. Math. Z 233, 287-303 (2000)

53. Schröer, J.: The Krull-Gabriel dimension of an algebra-open problems and conjectures. In: Infinite Length Modules, Trends Math., pp. 419-424. Birkhäuser, Basel (2000)

54. Skowroński, A.: Selfinjective algebras of polynomial growth. Math. Ann 285, 177-199 (1989)

55. Skowroński, A.: Algebras of polynomial growth. In: Topics in Algebra, Part 1, Banach Center Publ., vol. 26, pp. 535-568. PWN, Warsaw (1990)

56. Skowroński, A.: Regular Auslander-Reiten components containing directing modules. Proc. Amer. Math. Soc. 120, 19-26 (1994)

57. Skowroński, A.: Generalized standard Auslander-Reiten components. J. Math. Soc. Japan 46, 517-543 (1994)

58. Skowroński, A.: Minimal representation-infinite artin algebras. Math. Proc. Cambridge Philos. Soc. 116, 229-243 (1994)

59. Skowroński, A.: On the composition factors of periodic modules. J. London Math. Soc. 49, 477-492 (1994)

60. Skowroński, A.: Cycle-finite algebras. J. Pure Appl. Algebra 103, 105-116 (1995)

61. Skowroński, A.: Simply connected algebras of polynomial growth. Compositio Math 109, 99-133 (1997)

62. Skowroński, A.: Tame algebras with strongly simply connected Galois coverings. Colloq. Math. 72, 335-351 (1997)

63. Skowroński, A.: Tame quasi-tilted algebras. J. Algebra 203, 470-490 (1998)

64. Skowroński, A.: Selfinjective algebras: finite and tame type. In: Trends in Representation Theory of Algebras and Related Topics, Contemporary Mathematics, vol. 406, pp. 169-238. Amer. Math. Soc., Providence (2006)

65. Skowroński, A., Wenderlich, M.: Artin algebras with directing indecomposable projective modules. J. Algebra 165, 507-530 (1994)

66. Skowroński, A., Yamagata, K.: Galois coverings of selfinjective algebras by repetitive algebras. Trans. Amer. Math. Soc. 351, 715-734 (1999)

67. Skowyrski, A.: Cycle-finite algebras with almost all indecomposable modules of projective or injective dimension at most one. Colloq. Math. 132, 239-270 (2013)

68. Skowyrski, A.: A characterization of cycle-finite generalized double tilted algebras. J. Algebra 416, 124 (2014)

69. Wenderlich, M.: Krull dimension of strongly simply connected algebras. Bull. Polish Acad. Sci. Math. 44, 473-480 (1996)

70. Zhang, Y.B.: The structure of stable components. Canad. J. Math. 43, 652-672 (1991)

71. Ziegler, M.: Model theory of modules. Ann. Pure Appl. Logic 26, 149-213 (1984) 